\title{
A New Approach to Calculating Electrical Energy Losses on Power Lines with a New Improved Three-Mode Method
}

\author{
Emir ALIBAŠIĆ, Boris Fažo, Ivica PETROVIĆ
}

\begin{abstract}
Increasing the energy efficiency of the electricity sector is the commitment of all national transmission operators. One of the main goals is to reduce losses in electrical networks. Electrical networks are one of the most important parts of the electric grid. Delivery reliability and the quality of electricity directly depend on the electrical networks. It is well known that losses in the transmission network can be defined as the difference between the electricity taken and delivered. Determining the losses in overhead power lines has several basic tasks. One task is to determine the difference between calculated losses and actual losses, to determine the reason for the difference between calculated and actual losses, and to present the necessary data for taking measures to reduce losses. The second task is an estimation of the loss index for planning and formulation of various plans. The purpose of this paper is to present a new approach to reduce the difference between calculated and actual losses by modifying the method in three modes, or a new approach to calculating the loss index. The proposed method in this paper significantly reduces the error in relation to the classic threemode method, which represents the original contribution of this work.
\end{abstract}

Keywords: calculation; electrical energy losses; losses; power; three points method; transmission networks

\section{INTRODUCTION}

The lifetime of the power transmission lines is a long investment for the construction of new power transmission lines and it is expensive, so the losses on the power transmission lines take special care. Theoretical determination of losses in the electrical grid EG is an important economic indicator for the preparation of the (EG) development and management plan [1]. It is therefore very important to develop reliable and advanced methods of calculating electrical grid electricity losses and to present an accurate loss data of the electrical grid. In the paper [2], difficulties in obtaining the required measured quantities for determining losses were presented. References [3-4] include methods for calculating the losses in the transmission lines that are most commonly used in practice. Reducing the risk of selecting the wrong parameter increases the reliability of the method. Many factors affect the losses in the electrical grid, such as generation and consumption at a given moment, weather conditions [5], transmission line geometry [6] etc., and the calculations of the electrical grid transmission lines are run for a certain period [3-4]. Losses on the overhead power lines depend on voltage and current. The relationship between these losses is presented in the paper [7]. Voltagedependent losses do not depend on the electrical load as the current-dependent losses. Both types of losses depend on weather conditions [5]. In papers [8-11], methods of minimizing losses on transmission lines have been studied.

During the operation and running of the electrical grid, the electrical load can be classified in three modes: maximum mode, minimum mode, and normal operating mode $[3,12]$.

This article presents a modified loss-calculation method in transmission networks based on three modes, whose results are compared to real measured values of a single area. For the purposes of analysis, a MATLAB software package was used, in which the original method of calculating losses in transmission networks based on the three modes presented in [3] and the modified method proposed in this paper were programmed.

\section{ANALYTICAL DESCRIPTION OF THE METHOD}

The three-mode method performs the calculation of typical load flows in three points and continues to use it to obtain the corresponding average power loss under typical daily loads [12]. In practice, it has been shown that three types of daily power loss averages can represent total energy losses within three modes of operation. The purpose of the three-mode method is to determine the loss index over the whole period. The total loss index is obtained by integrating individual indices of losses in all three modes. Reference [3] describes the steps of calculating electricity losses based on three modes. The method proposed in this paper in relation to the method described in reference [3] differs in determining the load value for the minimum, maximum, and normal mode.

Calculation of flows with typical loads is carried out in three points or three features, to obtain the corresponding average loss of power under typical daily loads [4]. Obviously, the three types of daily average losses of the power may represent the total energy losses within the three operating modes. Line loss index on three points integrated to obtain an index of loss throughout the entire period is the basic concept of loss calculation in highvoltage grids based on the power losses in three points.

Theoretical calculation of losses for a long period that serves as a check is described in the next steps.

1) Application of the long period determined from the amount of energy in order to obtain the average load and the application of the measured maximum load to obtain factors of active power loads in long period $f_{p}$, and the minimum load index $\beta_{p}$, which gives the index of changes $1 / X$. Permitted medium power for three selected points are $P_{1 *}, P_{2^{*}}, P_{3^{*}}$, and then $P_{1^{*}}=\left(\beta_{p}+P_{s . m}\right) / 2, P_{2^{*}}=f_{p}, P_{3^{*}}=$ $\left(P_{m . b}+1,0\right) / 2$.

2) It is necessary to choose the typical curve of daily load for the three points, and provide them with daily maximum load, or $P_{1 \max }, P_{2 \max }, P_{3 \max }$. We also need to multiply real maximum load for the entire period up to the value per unit of average power. Compare the daily maximum load with the product and take $\left(P_{\Sigma \text {.max }} P_{1^{*}}\right) / P_{1 \max },\left(P_{\Sigma \text { max }} P_{2^{*}}\right) / P_{2 \max }$, $\left(P_{\Sigma_{\text {.max }}} P_{3^{*}}\right) / P_{3 \max }$, as coefficients of proportionality for 
adjusting to get a new distribution of the three points on typical daily load.

3) Performing calculated 24-hour power flows adjusted by distribution of three points on typical daily load, to obtain the average daily energy losses $\Delta P_{1} \%, \Delta P_{2} \%$ and $\triangle P_{3} \%$.

4) Using typical daily load curve for network in three ways to calculate the coefficients $K_{1}, K_{2}, K_{3}$ and using the formula (4), we calculate the losses indexes $\Delta A_{1} \%, \Delta A_{2} \%$ and $\triangle A_{3} \%$ according to three points of division.

5) With a combination of coefficients of low load factor that appears in most of the longer periods, we get the ratio between the electrical quantities of three shared characteristics $A_{1}: A_{2}: A_{3}$ and the ratio between the electrical quantities in three points of division of the total quantity of electricity $\alpha_{1}: \alpha_{2}: \alpha_{3}$.

6) Calculation of losses index for an entire period is obtained by the following formula:

$$
\Delta A_{\Sigma} \%=\frac{\Delta A_{\Sigma}}{A}=\frac{\left(\Delta A_{1} \% A_{1}+\Delta A_{2} \% A_{2}+\Delta A_{3} \% A_{3}\right)}{\left(A_{1}+A_{2}+A_{3}\right)}
$$

where $\Delta A_{1}, \Delta A_{2}$ and $\Delta A_{3}$ are loss indices divided into three modes.

Replacing the ratio of the average load, we get:

$$
\Delta A_{\Sigma} \%=0,233 \Delta A_{1} \%+0,660 \Delta A_{2} \%+0,107 \Delta A_{3} \%,
$$

Fig. 1 shows the flowchart and the new calculation method used for calculating losses.

According to formula (2), the total losses divided into three modes have a different degree of impact on the overall loss index: the normal mode has the greatest influence, followed by the minimum mode of operation, and ultimately the minimum impact has the maximum mode.
Determining the period, load and region

Calculation of parameters: $A, P_{a v}, P_{\min }, f, \beta, 1 / \lambda$

Calculating power points in three modes:

Determination of loss index change. Creating a table of losses, sorting and division into three modes. $P_{\text {s.av }}$, $P_{a v}, P_{\text {max. } . v}$ and $\Delta \boldsymbol{P}_{\boldsymbol{s}} \%, \Delta \boldsymbol{P}_{\boldsymbol{m}} \%, \Delta \boldsymbol{P}_{\boldsymbol{b}} \%$

Determination of the ratio of the amount of electrical energy for three modes. $\alpha_{1}, \alpha_{2}, \alpha_{3}$

energy for three modes. $\alpha_{1}, \alpha_{2}, \alpha_{3}$
$\Delta A_{s}, \Delta A_{m}, \Delta A_{b}$

Determination of total losses by loss index $\Delta P_{\Sigma} \%$, loss factor $F$, Curve shape coefficient $K$, loss index for the whole period $\Delta A_{\Sigma} \%$ and total losses $\Delta A_{\Sigma}$.

Figure 1 Flowchart of the new method of calculating losses

\section{AN EXAMPLE OF CALCULATION OF LOSSES IN THE TRANSMISSION NETWORK}

\subsection{An Example of calculating losses in the Transmission Network Based on Three Modes [3]}

The official data available on the NOS B\&H website Independent System Operator in Bosnia and Herzegovina www.nosbih.ba were used to present the method for calculating losses in three modes and a modified loss calculation method in three modes. The results were taken on the territory of Bosnia and Herzegovina for one typical day given in Tab. 1 .

\begin{tabular}{|c|c|c|c|c|c|c|c|c|}
\hline Hour & 1 & 2 & 3 & 4 & 5 & 6 & 7 & 8 \\
\hline Load (MW) & 1.110 & 1.026 & 989 & 971 & 993 & 1.069 & 1.270 & 1.474 \\
\hline Losses (MW) & 36 & 34 & 36 & 35 & 33 & 31 & 27 & 32 \\
\hline Hour & 9 & 10 & 11 & 12 & 13 & 14 & 15 & 16 \\
\hline Load (MW) & 1.554 & 1.565 & 1.521 & 1.503 & 1.478 & 1.465 & 1.488 & 1.466 \\
\hline Losses (MW) & 35 & 42 & 40 & 41 & 37 & 40 & 41 & 36 \\
\hline Hour & 17 & 18 & 19 & 20 & 21 & 22 & 23 & 24 \\
\hline Load (MW) & 1.461 & 1.463 & 1.602 & 1.672 & 1.615 & 1.509 & 1.353 & 1.206 \\
\hline Losses (MW) & 35 & 32 & 34 & 37 & 39 & 42 & 44 & 38 \\
\hline Total load (MW) & & 32.823 & & & & & & \\
\hline Total losses (MW) & & 877 & & & & & & \\
\hline
\end{tabular}

Table 1 Results of calculating losses in one region for a typical day

Fig. 2 shows the daily load diagram of the consumption of the analysed region used in this example. 1) Calculation of the total load curve parameters. The load points are:

$A=\sum_{1}^{24} P_{i} t=32823 \mathrm{MW}$

$$
\begin{aligned}
& P_{a v}=\frac{A}{24}=1367,62 \mathrm{MW}, \\
& P_{\max }=1672 \mathrm{MW}, P_{\min }=971 \mathrm{MW} \\
& f=\frac{P_{a v}}{P_{\max }}=0,818, \\
& \beta=\frac{P_{\min }}{P_{\max }}=0,58,
\end{aligned}
$$


$\frac{1}{\lambda}=\frac{(1-f)}{(f-\beta)}=0,765$

where: $f-$ is the load factor, $\beta-$ minimum load index, $1 / \lambda$ - load change index.

Daily power loss index is given:

$\Delta P \%=\frac{\sum \Delta P_{t}}{A}=\frac{877}{32823}=2,672 \%$

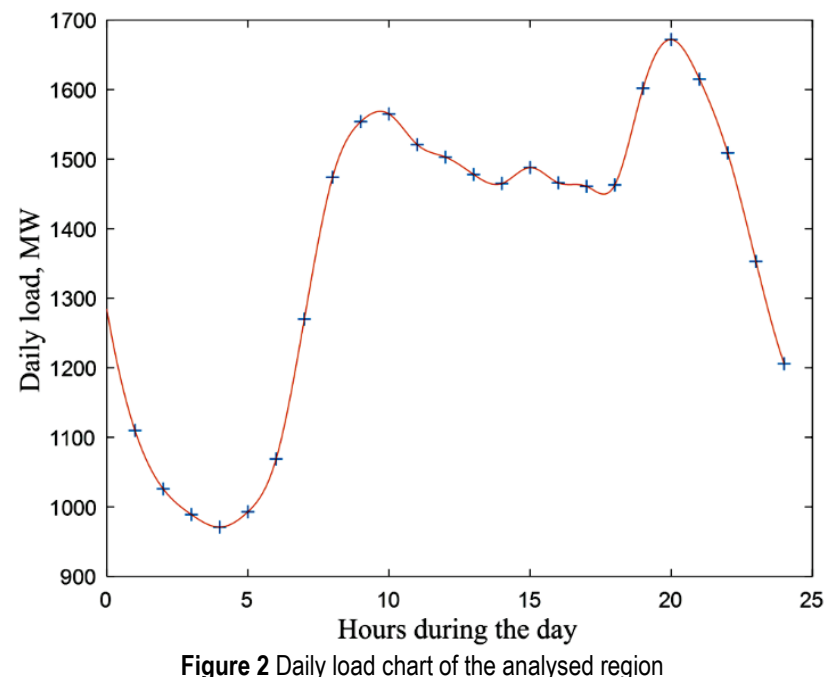

2) Calculation of power points in three modes: $f=0,818$. Using the formula for a combination of high load factor, we get:

$$
\begin{aligned}
& P_{s . m *}=0,008\left(\frac{1}{\lambda}\right)^{2}-0,1266\left(\frac{1}{\lambda}\right)+0,9043=0,812133 \\
& P_{s . m}=P_{s m *} \times P_{\max }= \\
& =0,812133 \times 1672 \times 10^{6}=1357,88 \mathrm{MW} \\
& P_{m . b *}=0,0052\left(\frac{1}{\lambda}\right)^{2}-0,0456\left(\frac{1}{\lambda}\right)+0,9913=0,95996 \\
& P_{m . b}=P_{m b *} \times P_{\max }= \\
& =0,95996 \times 1672 \times 10^{6}=1605,05 \mathrm{MW}
\end{aligned}
$$

3) Calculation of the average load of each divided mode.

Minimum mode:

$$
\begin{aligned}
& P_{\text {s.av }}=\frac{\left(\beta+P_{s m *}\right)}{2} \times P_{\max }= \\
& =\frac{(0,58+0,812133)}{2} \times 1672 \times 10^{6}=1163,82 \mathrm{MW}
\end{aligned}
$$

Maximum mode:

$$
\begin{aligned}
& P_{b . a v}=\frac{\left(P_{m .1}+1\right)}{2} \times P_{\max }= \\
& =\frac{(0,95996+1)}{2} \times 1672 \times 10^{6}=1638,53 \mathrm{MW}
\end{aligned}
$$

4) Change of the power loss index corresponding to the average load in three modes a) $P_{\text {s.av }}$ at the minimum operating mode corresponds to the power in 4 hours, and the change of the power loss index is:

$$
\Delta P_{s} \%=\Delta P_{4 *} \% \times\left(\frac{P_{s . a v}}{P_{4 *}}\right)^{2}=5,04 \%
$$

where: $\Delta P_{3^{*}}-$ is the ratio of power and load losses at $4 \mathrm{~h}$; $P_{\text {s.av }}-$ is the average minimum mode load; $P_{3^{*}}-$ is the measured load in $4 \mathrm{~h}$.

b) $P_{a v}$ in normal mode corresponds to the power at 8 hours, and so:

$\Delta P_{m} \%=\Delta P_{8 *} \% \times\left(\frac{P_{a v}}{P_{8 *}}\right)^{2}=1,87 \%$

where: $\Delta P_{8^{*}}-$ is the ratio of power and load losses at $8 \mathrm{~h}$; $P_{8^{*}}-$ is the measured load in $8 \mathrm{~h}$.

c) $P_{\text {max.av }}$ at maximum operating mode corresponds to power at $15 \mathrm{~h}$, and so:

$\Delta P_{b} \%=\Delta P_{20 *} \% \times\left(\frac{P_{b . a v}}{P_{20 *}}\right)^{2}=2,12 \%$

where: $P_{b . a v}-$ is the average maximum mode load; $P_{20 *}-$ is the measured load in $20 \mathrm{~h}$.

5) Calculation of the ratio of the amount of electrical energy for three modes. According to the integral operation of the load curve for the combination of high load index parameters when $1 / \lambda=0,6$, the ratio of the amount of electrical energy for three modes Tab. 3.4 from the literature [1] is $\alpha_{1}: \alpha_{2}: \alpha_{3}=1: 4,526: 1,655$; when $1 / \lambda=$ $0,8, \alpha_{1}: \alpha_{2}: \alpha_{3}=1: 4,80: 2,33$. According to the calculation using interpolation when $1 / \lambda=0.765$, the ratio of the amount of electrical energy for three modes is $\alpha_{1}$ : $\alpha_{2}: \alpha_{3}=1: 4,75: 2,19$.

6) The calculation of electrical energy losses is divided into three modes.

Minimum mode:

$\Delta A_{s}=\frac{A_{\Sigma} \times \alpha_{1}}{\alpha_{1}+\alpha_{2}+\alpha_{3}} \Delta P_{s} \%=208,76 \mathrm{MWh}$

Normal mode:

$\Delta A_{m}=\frac{A_{\Sigma} \times \alpha_{2}}{\alpha_{1}+\alpha_{2}+\alpha_{3}} \Delta P_{m} \%=367,20 \mathrm{MWh}$

Maximum mode:

$\Delta A_{b}=\frac{A_{\Sigma} \times \alpha_{3}}{\alpha_{1}+\alpha_{2}+\alpha_{3}} \Delta P_{b} \%=191,93 \mathrm{MWh}$

where: $\Delta P_{s} \%, \Delta P_{m} \%$ and $\Delta P_{b} \%$ - are power loss indices in minimum mode, normal and maximum mode. 
7) Calculation of total losses by the loss index for a whole period.

The power loss index for a whole period is:

$\Delta P_{\Sigma} \%=\frac{877}{32823}=2,672 \%$

The loss factor is calculated by means of coefficients $f$ and $\beta$

$F=0,639 f^{2}+0,361(f+f \beta-\beta)=0,6848$

The coefficient of the curve shape of the total load is:

$K=\frac{\sqrt{F}}{f}=\frac{\sqrt{0,6848}}{0,818}=1,012$

Using the formula for calculating the index of electrical energy losses for a full-time period we get:

$\Delta A_{\Sigma} \%=K \Delta P_{\Sigma} \%=1,012 \times 2,672 \%=2,704 \%$

$\Delta A_{\Sigma}=A \Delta A_{\Sigma} \%=32823 \times 10^{6} \times 2,704 \%=887,53 \mathrm{MWh}$

Error method calculation. There was an error between the loss of electrical energy by dividing into three modes and the results obtained by measuring the loss index, and so:

$$
\Delta A_{\Sigma}^{\prime}=\Delta A_{s}+\Delta A_{m}+\Delta A_{b}=767,89 \mathrm{MWh}
$$

where: $\Delta A_{s}, \Delta A_{m}$ and $\Delta A_{b}$ - are the losses of the electrical energy of minimum, normal and maximum mode.

The relative error is:

$\delta \Delta A_{\Sigma}=\frac{\left(\Delta A_{\Sigma}-\Delta A_{\Sigma}^{\prime}\right)}{\Delta A_{\Sigma}} \times 100 \%=13,48 \%$

where: $\Delta A_{\Sigma}^{\prime}-$ is total electrical energy losses divided into three modes.

\subsection{An Example of the Calculation of Losses in the Transmission Network with an Improved Three-Mode Method}

Tab. 1 shows the loads and the estimated losses per hour of a typical day in one region.

1) Calculation of total load curve parameters. The load points are:

$$
\begin{aligned}
& A=\sum_{1}^{24} P_{i} t=32823 \mathrm{MW}, \\
& P_{a v}=\frac{A}{24}=1367,62 \mathrm{MW}, \\
& P_{\max }=1672 \mathrm{MW}, P_{\min }=971 \mathrm{MW}
\end{aligned}
$$

$f=\frac{P_{a v}}{P_{\max }}=0,818$

$\beta=\frac{P_{\min }}{P_{\max }}=0,58$,

$\frac{1}{\lambda}=\frac{(1-f)}{(f-\beta)}=0,765$,

where: where: $f$ - is the load factor, $\beta$ - minimum load index, $1 / \lambda$ - load change index.

Daily power loss index is:

$$
\Delta P \%=\frac{\sum \Delta P_{t}}{A}=\frac{877}{32823}=2,672 \% .
$$

2) Calculation of power points in three modes: $f=0,818$. Using the formula for a combination of high load factor, we get:

$$
\begin{aligned}
& P_{s . m *}=0,008\left(\frac{1}{\lambda}\right)^{2}-0,1266\left(\frac{1}{\lambda}\right)+0,9043=0,812133 \\
& P_{s . m}=P_{s m *} \times P_{\max }= \\
& =0,812133 \times 1672 \times 10^{6}=1357,88 \mathrm{MW} \\
& P_{m . b *}=0,0052\left(\frac{1}{\lambda}\right)^{2}-0,0456\left(\frac{1}{\lambda}\right)+0,9913=0,95996 \\
& P_{m . b}=P_{m b *} \times P_{\max }= \\
& =0,95996 \times 1672 \times 10^{6}=1605,05 \mathrm{MW}
\end{aligned}
$$

3) Calculation of the average load of each divided mode.

Minimum mode:

$$
\begin{aligned}
& P_{\text {s.av }}=\frac{\left(\beta+P_{s m^{*}}\right)}{2} \times P_{\max }= \\
& =\frac{(0,58+0,812133)}{2} \times 1672 \times 10^{6}=1163,82 \mathrm{MW}
\end{aligned}
$$

Maximum mode:

$$
\begin{aligned}
& P_{b . a v}=\frac{\left(P_{m .1}+1\right)}{2} \times P_{\max }= \\
& =\frac{(0,95996+1)}{2} \times 1672 \times 10^{6}=1638,53 \mathrm{MW}
\end{aligned}
$$

4) Change of the power loss index corresponding to the average load in three modes.

To determine the power corresponding to particular mode (minimum, normal, and maximum) from Tab. 1, we form Tab. 2 in order to sort the load values from the lowest to the highest.

a) $P_{\text {s.av }}$ in minimum operating mode corresponds to the power value of $1079,25 \mathrm{MV}$, and the change of the power loss index is:

$$
\Delta P_{S} \%=\Delta P_{\min *} \% \times\left(\frac{P_{\text {s.av }}}{P_{\min *}}\right)^{2}=3,62 \%
$$


where: $\Delta P_{\text {min* }}-$ is the ratio of power and load losses in minimum mode; $P_{\text {s.av }}$ - is the average minimum mode load; $P_{\text {min* }}-$ is the average load corresponding to the minimum mode according to Tab. 2 .

Table 2 Losses in one region sorted by load, calculated average values of losses and loads corresponding to each mode

\begin{tabular}{|c|c|c|c|c|c|}
\hline Hour & $\begin{array}{l}\text { Losses } \\
(\mathrm{MW})\end{array}$ & $\begin{array}{l}\text { Load } \\
(\mathrm{MW})\end{array}$ & $\begin{array}{c}\text { Losses - average } \\
\text { (MW) }\end{array}$ & $\begin{array}{c}\text { Load - average } \\
\text { (MW) }\end{array}$ & Mode \\
\hline 4 & 35 & 971 & \multirow{8}{*}{33,75} & \multirow{8}{*}{1079,25} & \multirow{8}{*}{$\begin{array}{l}\text { ठ } \\
\Xi \\
\Xi \\
\Xi \\
\Xi \\
\Xi \\
. \Xi \\
\Xi\end{array}$} \\
\hline 3 & 36 & 989 & & & \\
\hline 5 & 33 & 993 & & & \\
\hline 2 & 34 & 1026 & & & \\
\hline 6 & 31 & 1069 & & & \\
\hline 1 & 36 & 1110 & & & \\
\hline 24 & 38 & 1206 & & & \\
\hline 7 & 27 & 1270 & & & \\
\hline 23 & 44 & 1353 & \multirow{8}{*}{37,125} & \multirow{8}{*}{1456} & \multirow{8}{*}{ 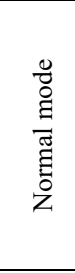 } \\
\hline 17 & 35 & 1461 & & & \\
\hline 18 & 32 & 1463 & & & \\
\hline 14 & 40 & 1465 & & & \\
\hline 16 & 36 & 1466 & & & \\
\hline 8 & 32 & 1474 & & & \\
\hline 13 & 37 & 1478 & & & \\
\hline 15 & 41 & 1488 & & & \\
\hline 12 & 41 & 1503 & \multirow{8}{*}{38,75} & \multirow{8}{*}{1567,625} & \multirow{8}{*}{ 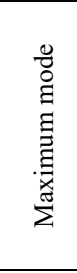 } \\
\hline 22 & 42 & 1509 & & & \\
\hline 11 & 40 & 1521 & & & \\
\hline 9 & 35 & 1554 & & & \\
\hline 10 & 42 & 1565 & & & \\
\hline 19 & 34 & 1602 & & & \\
\hline 21 & 39 & 1615 & & & \\
\hline 20 & 37 & 1672 & & & \\
\hline
\end{tabular}

b) $P_{a v}$ in normal operating mode corresponds to a power of $1456 \mathrm{MW}$, and so:

$$
\Delta P_{m} \%=\Delta P_{\text {norm } *} \% \times\left(\frac{P_{a v}}{P_{\text {norm } *}}\right)^{2}=2,25 \%
$$

where: $\Delta P_{\text {norm* }}-$ is the ratio of power and load losses determined for normal mode according to Tab. 2 ; $P_{\text {norm* }}-$ the load determined for normal mode according to Tab. 2 .

c) $P_{\text {max.av }}$ in maximum operating mode corresponds to the power of $1567,625 \mathrm{MW}$, and so:

$\Delta P_{b} \%=\Delta P_{\max *} \% \times\left(\frac{P_{b . a v}}{P_{\max *}}\right)^{2}=2,70 \%$

where: $\Delta P_{\text {max* }}$ - is the ratio of power and load losses determined for maximum mode according to Tab. $2 ; P_{b . a v}$ - average maximum mode load; $P_{\text {max* }}$ - the load determined for maximum mode according to Tab. 2 .

5) Calculation of the ratio of the amount of electrical energy for three modes. According to the integral operation from the load curve for the combination of high load index parameters when $1 / \lambda=0,6$, the ratio of the amount of electrical energy for three modes Tab. 3.4 from the literature [1] is $\alpha_{1}: \alpha_{2}: \alpha_{3}=1: 4,526: 1,655$; when $1 / \lambda=$ $0,8, \alpha_{1}: \alpha_{2}: \alpha_{3}=1: 4,80: 2,33$. According to the calculation using interpolation when $1 / \lambda=0.765$, the ratio of the amount of electrical energy for three modes is $\alpha_{1}$ : $\alpha_{2}: \alpha_{3}=1: 4,75: 2,19$.
6) The calculation of electrical energy losses is divided into three modes.

Minimum mode:

$$
\Delta A_{s}=\frac{A_{\Sigma} \times \alpha_{1}}{\alpha_{1}+\alpha_{2}+\alpha_{3}} \Delta P_{s} \%=149,65 \mathrm{MWh}
$$

Normal mode:

$$
\Delta A_{m}=\frac{A_{\Sigma} \times \alpha_{2}}{\alpha_{1}+\alpha_{2}+\alpha_{3}} \Delta P_{m} \%=441,81 \mathrm{MWh}
$$

Maximum mode:

$$
\Delta A_{b}=\frac{A_{\Sigma} \times \alpha_{3}}{\alpha_{1}+\alpha_{2}+\alpha_{3}} \Delta P_{b} \%=244,44 \mathrm{MWh}
$$

where: $\Delta P_{s} \%, \Delta P_{m} \%$ and $\Delta P_{b} \%$ - are power loss indices in minimum mode, normal and maximum mode.

7) Calculation of total losses by loss index for a whole period.

The power loss index for a whole period is:

$$
\Delta P_{\Sigma} \%=\frac{877}{32823}=2,672 \%
$$

The loss factor is calculated by means of coefficients $f$ and $\beta$

$$
F=0,639 f^{2}+0,361(f+f \beta-\beta)=0,6848
$$

The coefficient of the curve shape of total loads is:

$$
K=\frac{\sqrt{F}}{f}=\frac{\sqrt{0,6848}}{0,818}=1,012
$$

Using the formula for calculating the index electrical energy losses for a full time period we get:

$\Delta A_{\Sigma} \%=K \Delta P_{\Sigma} \%=1,012 \times 2,672 \%=2,704 \%$

$\Delta A_{\Sigma}=A \Delta A_{\Sigma} \%=32823 \times 10^{6} \times 2,704 \%=887,53 \mathrm{MWh}$

Error method calculation. There was an error between the loss of electrical energy by dividing into three modes and the results obtained by measuring the loss index, and so:

$$
\Delta A_{\Sigma}^{\prime}=\Delta A_{s}+\Delta A_{m}+\Delta A_{b}=835,90 \mathrm{MWh}
$$

where: $\Delta A_{s}, \Delta A_{m}$ and $\Delta A_{b}$ - are the losses of the electrical energy of minimum, normal and maximum mode.

The relative error is:

$$
\delta \Delta A_{\Sigma}=\frac{\left(\Delta A_{\Sigma}-\Delta A_{\Sigma}^{\prime}\right)}{\Delta A_{\Sigma}} \times 100 \%=5,817 \%
$$


where: $\Delta A_{\Sigma}^{\prime}-$ is total electrical energy losses divided into three modes.

The proposed method significantly reduces the error with respect to the previously presented, which is the original contribution of this work. It is obvious that in the equation number 27 of the three modes, the relative error is $13.47 \%$, while the relative error of the proposed modification of the three modes method is $5.817 \%$, equation 52.

With this proposed method, the calculations are performed with considerably lower error values.

\section{CALCULATION OF LOSSES IN THE TRANSMISSION NETWORK USING MATLAB TOOLS}

For this paper and verification of the proposed improvement of the method, the MATLAB R2017b software package was used. The method was programmed as a "m" file and an estimation was calculated for several typical days of some regions.

Example

For the first example, an illustrated example in practice is presented [4] and is shown below in Tab. 3 .

Table 3 Results of loss calculations in one region

\begin{tabular}{|c|c|c|c|c|c|c|c|c|}
\hline Hour & 1 & 2 & 3 & 4 & 5 & 6 & 7 & 8 \\
\hline Load (MW) & 195 & 191 & 189 & 190 & 193 & 205 & 212 & 220 \\
\hline Losses (MW) & 1,35 & 1,25 & 1,25 & 1,25 & 1,30 & 1,55 & 1,65 & 1,85 \\
\hline Hour & 9 & 10 & 11 & 12 & 13 & 14 & 15 & 16 \\
\hline Load (MW) & 229 & 240 & 234 & 214 & 223 & 233 & 236 & 239 \\
\hline Losses (MW) & 2,15 & 2,50 & 2,45 & 1,75 & 2,00 & 2,30 & 2,40 & 2,50 \\
\hline Hour & 17 & 18 & 19 & 20 & 21 & 22 & 23 & 24 \\
\hline Load (MW) & 237 & 222 & 229 & 237 & 232 & 222 & 209 & 212 \\
\hline Losses (MW) & 2,47 & 1,97 & 2,17 & 2,45 & 2,25 & 1,95 & 1,65 & 1,30 \\
\hline Total load (MW) & & 5.243 & & & & & & \\
\hline Total losses (MW) & & 45,71 & & & & & & \\
\hline
\end{tabular}

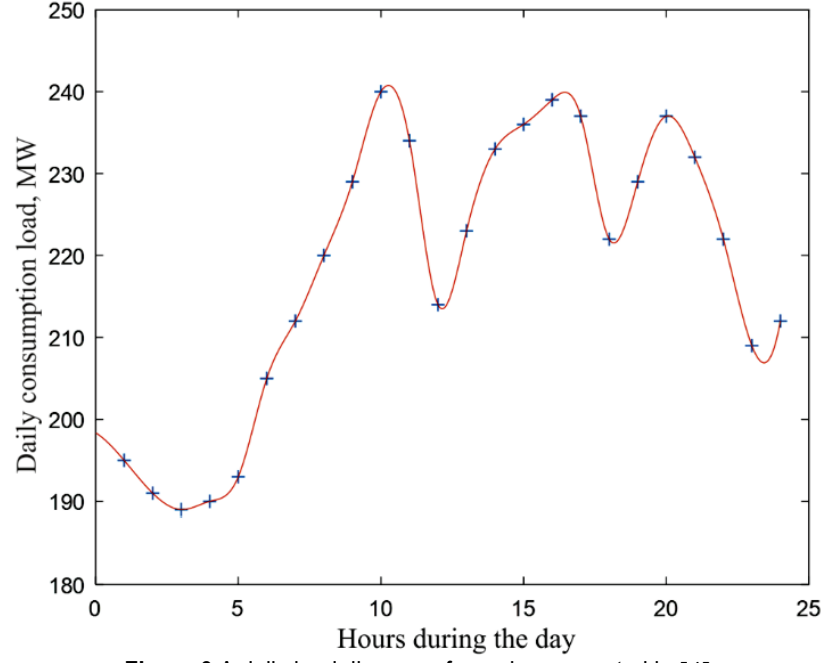

Figure $3 \mathrm{~A}$ daily load diagram of a region presented in [4]

Table 4 Comparison of the results of the examples presented in [4] and the results obtained by the modified three-mode method for one region

\begin{tabular}{|c|c|c|}
\hline Value - Term & $\begin{array}{c}\text { Results Presented } \\
\text { in [4] }\end{array}$ & $\begin{array}{c}\text { Results obtained } \\
\text { by the modified } \\
\text { method }\end{array}$ \\
\hline $\begin{array}{c}\text { Change of minimum mode } \\
\text { loss index }\end{array}$ & $0,686 \%$ & $0,672 \%$ \\
\hline $\begin{array}{c}\text { Change of normal mode loss } \\
\text { index }\end{array}$ & $0,8295 \%$ & $0,831 \%$ \\
\hline $\begin{array}{c}\text { Change of maximum mode } \\
\text { loss index }\end{array}$ & $0,98 \%$ & $1,020 \%$ \\
\hline $\begin{array}{c}\text { Calculation of total losses by } \\
\text { the loss index for a whole } \\
\text { period }\end{array}$ & $45.771,39 \mathrm{kWh}$ & $45.771,39 \mathrm{kWh}$ \\
\hline $\begin{array}{c}\text { Calculation of energy losses } \\
\text { by dividing into three modes }\end{array}$ & $44.637,00 \mathrm{kWh}$ & $45.263,82 \mathrm{kWh}$ \\
\hline Relative Error & $2,480 \%$ & $1,109 \%$ \\
\hline
\end{tabular}

The representation of the daily consumption load diagram of the analyzed region used in this case is given in Fig. 3.

To correctly evaluate and valorise data, it is necessary to compare the results according to the two methods mentioned above. In Tab. 4, a summary of the features of the method and the comparison of the results is given.

From Tab. 4 it is noticeable that in the first method the relative error of the calculation of losses is $2.48 \%$, and that the relative error in the modified loss calculation method is considerably smaller and is approximately $1.11 \%$.

\section{CONCLUSION}

New approaches to calculation of high-voltage transmission network losses are expressed through a number of advantages. The steady increase in energy consumption and the physical constraints of transmission lines initiate the need for the development of new methods and loss detection tools on the transmission network.

The results of the analysis presented in this paper were obtained based on the actual measured results and the results of the calculation, using the three modes method and the modified three-mode method. Verification of the methods in this paper was carried out through two examples. The first example is the transmission network of Bosnia and Herzegovina and the second example was used in [4]. By using the proposed approach to determine the loss index for the three-mode approach, the relative error is significantly reduced of the existing method and the calculation of the losses. In addition to reducing the relative error in the calculation, the advantage of this method is also seen through increased reliability, which significantly contributes to the improvement of the existing methods and the overall real estimate. The proposed approach eliminates a very sensitive input parameter, and thus an error when determining it.

\section{REFERENCES}

[1] Han, S., Li, K., \& Xu, K. (2016). An online calculation method of theoretical power losses for high-voltage distribution system based on rapid modelling and data quality analysis. Proceedings of 2016 IEEE International 
Conference on Mechatronics and Automation. August 7-10, Harbin, China. https://doi.org/10.1109//CMA.2016.7558667

[2] Chen, Y. \& Chang, Q. (1999). Study of GPS-based real-time loss calculation of electric power system. Power System Technology, 23(12), 24-27.

[3] Wu, A. \& Ni, B. (2016). Line loss analysis and calculation of electric power systems. China Electric Power Press. https://doi.org/10.1002/9781118867273

[4] Petrović, I., Mikulić, F., Baus, Z., Nikolovski, S., \& Galić, D. (2018). A New Approach for the Analysis of Losses in Calculation of High-Voltage Transmission Networks. Tehnički vjesnik, 25(5), 1506-1512. https://doi.org/10.17559/TV-20170206144804

[5] Baran I., Costea M., \& Tudor, L. (2017). Power Losses on Overhead Lines under Various Loading Regimes and Weather Conditions. IEEE, University "Politehnica" of Bucharest. Bucharest, Romania. https://doi.org/10.1109/ISEEE.2017.8170656

[6] Ahmadi, H. \& Armstrong, M. (2016). Transmission Line Impedance Calculation Using Detailed Line Geometry and HEM Soil Resistivity Measurements. IEEE Canadian Conference on Electrical and Computer Engineering (CCECE). https://doi.org/10.1109/CCECE.2016.7726808

[7] Al-Hamouz, Z. M. (2019). Corona power loss versus ohmic power loss in HYDC transmission lines. Department of Electrical Engineering. King Fahd University of Petroleum and Minerals, Dhahran 31261, Saudi Arabia.

[8] Ramesh, L., Chowdhury, S. P., Chowdhury, S., Natarajan, A. A., \& Gaunt, C. T. (2009). Minimization of power loss in distribution networks by different techniques. International Journal of Electrical Power and Energy Systems Engineering, 2(1), 1-6.

[9] Rugthaicharoencheep, N. \& Sirisumrannukul, S. (2009). Feeder reconfiguration for loss reduction in distribution system with distributed generators by Tabu Search. GMSARN International Journal, 3, 47-54.

[10] Sinsuphun, N., Leeton, U., Kwannetr, D., Uthitsunthorn, U., \& Kulworawanichpong, T. (2011). Loss minimization using optimal power flow based on swarm intelligences. Transactions on Electrical Engineering, Electronics, and Communications, 9(1), 212-222.

[11] Bamigbola, O. M., Ali, M. M., \& Oke, M. O. (2014). Mathematical modelling of electric power flow and the minimization of power losses on power transmission lines Applied Mathematics and Computation, 241, 214-221. https://doi.org/10.1016/j.amc.2014.05.039

[12] Wu, A. (2011). Three-mode division of loads under multiplying probability assumption and its application. Distribution and Utilization, 28(2), 1-4.

[13] Fekete, K., Nikolovski, S., \& Baus, Z. (2007). Simulation of Power Flows in Croatian Power System. The $6^{\text {th }}$ EUROSIM Congress. Ljubljana, Slovenia. Sept. 9-13.

[14] Nikolovski, S., Marić, P., \& Baus, Z. (2007). Electromagnetic Field Calculation of Transformer Station 400/110 kV Ernestinovo Using CDEGS Software. Journal of Electrical Engineering, 58(4), 207-213.

[15] Klaić, Z., Damir, Š., \& Baus, Z. (2011). Probability Density Functions of Voltage Sags Measured Indices. Journal of Electrical Engineering, 62(6). https://doi.org/10.2478/v10187-011-0053-8

\section{Contact information:}

Emir ALIBAŠIĆ, dipl. ing. el.

ZZO TK, BiH,

Franjevačka 36, 75000 Tuzla, Bosnia and Herzegovina emir.alibasic@gmail.com

Boris FAŽo, mag ing. el.

Ivica PETROVIĆ, PhD,

Croatian Transmission System Operato

Network Center Osijek, Osijek, Croatia 